\title{
Transcatheter aortic valve implantation through a transcarotid approach and cerebral injury
}

\author{
mann \\ Miłosz Jaguszewski ${ }^{4}$, Jan Rogowski ${ }^{1}$, Edyta Szurowska ${ }^{3}$, Dariusz Jagielak ${ }^{1}$ \\ 1 Department of Cardiac and Vascular Surgery, Medical University of Gdańsk, Gdańsk, Poland \\ 2 Department of Quality of Life Research, Medical University of Gdańsk, Gdańsk, Poland \\ 3 Department of Radiology, Medical University of Gdańsk, Gdańsk, Poland \\ 4 1st Department of Cardiology, Medical University of Gdańsk, Gdańsk, Poland
}

Correspondence to: Aleksandra Stańska, MSc, Department of Cardiac and Vascular Surgery, Medical University of Gdańsk, ul. Smoluchowskiego 17, 80-214 Gdańsk, Poland, phone: +48585844200; email: astanska@gumed.edu.pl Received: January 24, 2020. Revision accepted: March 22, 2020.

Published online: March 30, 2020. Kardiol Pol. 2020; 78 (7-8): 756-758 doi:10.33963/KP.15267

Copyright by the Author(s), 2020
Introduction In 2010, the first case of transcatheter aortic valve implantation (TAVI) via the left carotid artery was presented in literature. Modine et $\mathrm{al}^{1}$ described the method as "the last resort" in case of unavailability of other possible approaches and pointed out that it should be preceded by a careful cerebral arterial assessment.

Until today, the great majority of procedures are still performed through a transfemoral approach. Of note, transfemoral TAVI may be obtained with a complete percutaneous approach or surgical access, which both have been shown to have similar safety and efficacy. ${ }^{2}$ Due to particular circumstances such as severe peripheral atherosclerosis and calcified vessels, significant descending aortic disease or physiological abnormalities of the vasculature, this pathway cannot always be performed and may not be the best option for the patient. On the other hand, transthoracic approaches are well known to be associated with higher mortality, ${ }^{3}$ therefore carotid access was suggested as the promising alternative.

Unfortunately, manipulation of the catheter in the carotid artery may be associated with an increased risk of cerebral injury. Nevertheless, the analysis of literature showed that the transcarotid TAVI procedure is similar to the transfemoral approach for mortality and morbidity. ${ }^{4,5}$ Moreover, the prevalence of neurological injury in transcarotid TAVI does not differ significantly from the existing data on transfemoral access. ${ }^{6,7}$ The chances are that the transcarotid TAVI procedure may be a propitious and attractive alternative to the current gold standard.
Is the risk of cerebral injury following transcarotid TAVI as high as it was claimed before? To answer this question, we conducted a single-center, observational preliminary study. The objective was to compare brain magnetic resonance imaging (MRI) scans in patients undergoing TAVI through transfemoral and transcarotid approaches and assess their declared quality of life and cognitive functioning in a 3-month follow-up.

Methods A nonrandomized study included 8 consecutive patients with severe, symptomatic aortic stenosis. Patients disqualified from the transfemoral access were considered for transcarotid TAVI. Patients with existing contraindications for MRI were excluded from the study.

Five patients underwent transfemoral TAVI and 3 patients underwent TAVI via carotid access. The process of qualification included physical examination, computed tomography angiography, and an interview with the patient and his relatives. Carotid Doppler was performed in all patients disqualified from the transfemoral approach to assess the possibility of an alternative access. The mean (SD) age in both groups was 83 (3.16) years; all patients had comparable comorbidities. In the transcarotid group, 2 out of 3 patients and in the transfemoral group, 1 out of 5 patients presented chronic atrial fibrillation. The mean Society of Thoracic Surgeons risk score for both groups was $9.9 \%$, and for transfemoral and transcarotid TAVI, 8.98\% vs $11.44 \%$, respectively.

All TAVI procedures were performed by a local Heart Team in a hybrid operating room equipped with a heart-lung machine on stand-by. The 

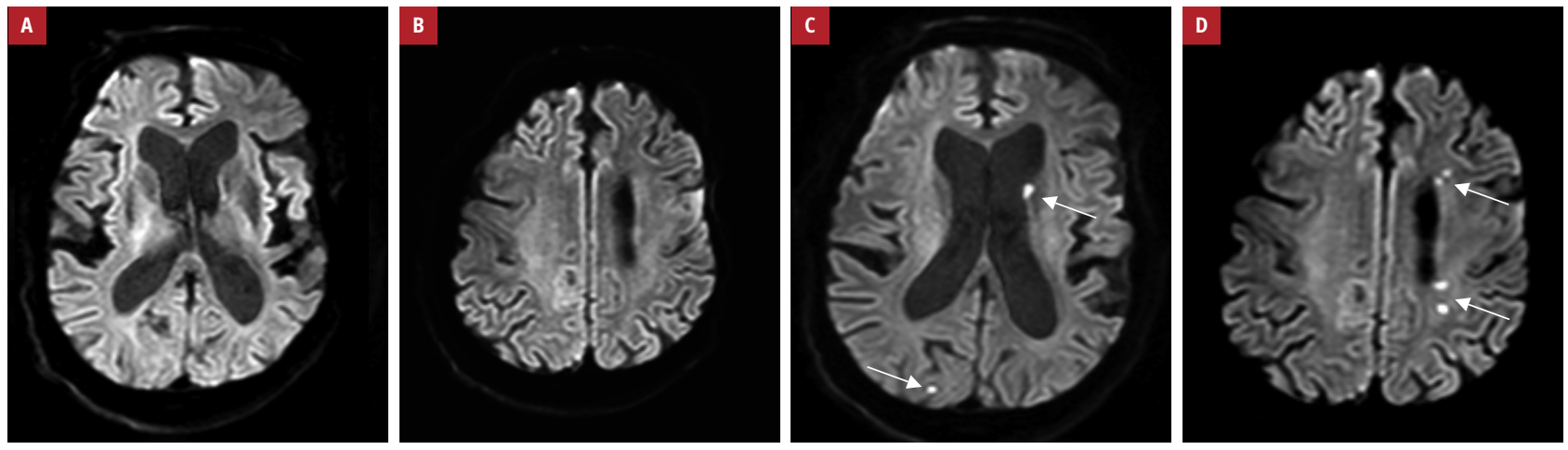

FIGURE 1 Diffusion-weighted magnetic resonance imaging: A, B - no acute ischemic brain lesions before transfemoral transcatheter aortic valve implantation;

C, D - acute ischemic brain lesions in the head of the caudate nucleus and in the left brain hemisphere (arrows) after transfemoral transcatheter aortic valve implantation

Evolut R valve (Medtronic, Minneapolis, Minnesota, United States) was implanted in all patients.

Transcarotid implantations were performed under general anesthesia using the left carotid artery. In order to expose the carotid artery, an approximately 5 -cm vertical incision above the clavicle was performed.

Transfemoral implantations were done under local anesthesia without any sedation. Vascular access was performed percutaneously and in all cases, percutaneous vascular closure devices were used.

In both groups, the position of the prosthesis was confirmed using a contrast medium. A further bolus of contrast medium was given after the procedure to evaluate the position of the valve and to estimate the eventual paravalvular leak and coronary ostia. After estimating the shape of the valve and paravalvular leak, the decision whether to perform post-dilatation was made.

Furthermore, all patients underwent noncontrast magnetic resonance brain imaging before and 3 to 5 days after the procedure, in order to assess the number of potential recent ischemic lesions.

All patients were able to and signed an informed consent form. The study design was approved by the Bioethics Committee of the Medical University of Gdańsk.

Statistical analysis The statistical analysis was performed using STATISTICA 12.0 PL (StatSoft, Kraków, Poland), yet due to the small number of patients, the analysis was not presented. Moreover, the analysis included basic statistical concepts such as means, medians (SD).

Results and discussion The procedures were performed without any complications and the mean hospital stay for both groups was approximately 5 days. Echocardiographic parameters were improving in comparison with baseline values.

In the transfemoral group, 3 patients' MRI scans showed several minor recent ischemic lesions, up to $7 \mathrm{~mm}$, in both hemispheres and the cerebellum (FIGURE 1A-1D). There were no recent lesions in the following 2 patients' scans. In the transcarotid group, there were no lesions found after the procedure, except for one patient with a single acute 4-mm ischemic lesion in the precentral gyrus, which was not significant clinically. The lesion was located in the left hemisphere, ipsilaterally to the used carotid artery. Three patients with lesions from the transfemoral group presented mild post-operative delirium symptoms, approximately 3 days after TAVI. One of them required the administration of antipsychotic medication.

Furthermore, the quality of life was evaluated using the Polish version of the EQ-5D-3L questionnaire and the screening of cognitive functioning was assessed with the Montreal Cognitive Assessment. The follow-up evaluation was performed after 3 months from the procedure in an outpatient clinic setting. The great majority of patients in both groups declared a significant improvement in the quality of life, including pain or discomfort and depression or anxiety symptoms. The screening of cognitive functioning did not show any significant results. $\mathrm{Pa}$ tient scores were minimally lower or similar to the baseline results.

The presented preliminary study compared brain MRI scans of 2 groups of patients who underwent TAVI through transfemoral and transcarotid approaches. None of acute ischemic lesions had any significant clinical consequences on the patients' wellbeing in the 3-month follow-up. Moreover, chronic atrial fibrillation did not seem to be a risk factor of ischemia following TAVI. Taking into account the Valve Academic Research Consortium 2 definitions of stroke, all patients with cerebral ischemic lesions underwent nondisabling stroke (modified Rankin Scale; $\mathrm{mRS}<2$ at 90 days or no increase in $\mathrm{mRS} \geq 1$ from the prestroke baseline). ${ }^{8}$

The preliminary data may suggest that patients undergoing TAVI in both described approaches are not exposed to severe cerebral injury. Therefore, 
we should consider transcarotid access as a promising alternative to transfemoral approach. First of all, transcarotid pathway presents a direct and significantly shorter route to the aortic valve from the entry. The additional benefit is an improved movement precision of catheter delivery.

Furthermore, the role of patient qualification is integral to the procedure's success. All patients considered for transcarotid access should undergo a detailed interview and transcarotid Doppler. Moreover, the assessment of calcium score should be an important part of computed tomography angiography in every patient. It can potentially be associated with higher risk of ischemic injury in TAVI. If the score raises doubts, it may be more adequate to choose nonvascular type of access and / or to use neuroprotective devices. The role of pre- and post-dilatation may be also connected with potential ischemic lesions, regardless of the type of access. Further, we have to reconsider the method of evaluating cognitive functioning and quality of life to make it more suitable and sensitive for this specific group of elderly patients.

Summarizing, despite the acute, mainly not significant clinically ischemic lesions, the quality of life was improving in both types of access. These preliminary results suggest that it is very possible that transcarotid access is safe and not connected with a higher risk of neurological injury. Further studies are needed to prove that hypothesis.

\section{ARTICLE INFORMATION}

CONFLICT OF INTEREST None provided

OPEN ACCESS This is an Open Access article distributed under the terms of the Creative Commons Attribution-Non Commercial-No Derivatives $4.0 \mathrm{In}$ ternational License (CC BY-NC-ND 4.0), allowing third parties to download articles and share them with others, provided the original work is properly cited, not changed in any way, distributed under the same license, and used for noncommercial purposes only. For commercial use, please contact the journal office at kardiologiapolska@ptkardio.pl.

HOW TO CITE Stańska A, Fijałkowska J, Targoński R, et al. Transcatheter aortic valve implantation through a transcarotid approach and cerebral injury. Kardiol Pol. 2020; 78; 756-758. doi:10.33963/KP.15267

\section{REFERENCES}

1 Modine T, Lemesle G, Azzaoui R, et al. Aortic valve implantation with the CoreValve ReValving System via left carotid artery access: first case report. J Thorac Cardiovasc Surg. 2010; 140: 928-929.

2 Kochman J, Kołtowski $k$, Huczek Z, et al. Complete percutaneous approach versus surgical access in transfemoral transcatheter aortic valve implantation: results from a multicentre registry. Kardiol Pol. 2018; 76: 202-208.

3 Koifman E, Magalhaes M, Kiramijyan S, et al. Impact of transfemoral versus transapical access on mortality among patients with severe aortic stenosis undergoing transcatheter aortic valve replacement. Cardiovasc Revasc Med. 2016; 17: 318-321.

4 Mylotte D, Sudre A, Teiger E, et al. Transcarotid transcatheter aortic valve replacement: feasibility and safety. JACC Cardiovasc Interv. 2016; 9: 472-480.

5 Wee IJY, Stonier T, Harrison M, et al. Transcarotid transcatheter aortic valve implantation: a systematic review. J Cardiol. 2018; 71: 525-533.

6 Overtchouk P, Alqdeimat I, Coisne A, et al. Transcarotid approach for TAVI: an optimal alternative to the transfemoral gold standard. Ann Cardiothorac Surg. 2017; 6: 555-557.

7 Nombela-Franco L, Webb JG, de Jaegere PP, et al. Timing, predictive factors, and prognostic value of cerebrovascular events in a large cohort of patients undergoing transcatheter aortic valve implantation. Circulation. 2012; 126: 3041-3053.

8 Lansky A, Ghare MI, Tchétché D, et al. TAVI and the brain: update on definitions, evidence of neuroprotection and adjunctive pharmacotherapy. EuroIntervention. 2018; 14: AB53-AB63. 